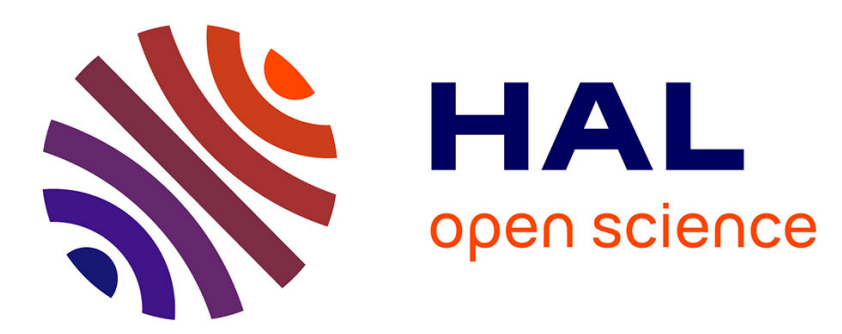

\title{
Investigation of elastic modes propagating in multi-wire helical waveguides
}

Fabien Treyssede, Laurent Laguerre

\section{To cite this version:}

Fabien Treyssede, Laurent Laguerre. Investigation of elastic modes propagating in multi-wire helical waveguides. Journal of Sound and Vibration, 2010, 329 (10), pp 1702-1716. hal-00612109

\section{HAL Id: hal-00612109 \\ https://hal.science/hal-00612109}

Submitted on 28 Jul 2011

HAL is a multi-disciplinary open access archive for the deposit and dissemination of scientific research documents, whether they are published or not. The documents may come from teaching and research institutions in France or abroad, or from public or private research centers.
L'archive ouverte pluridisciplinaire HAL, est destinée au dépôt et à la diffusion de documents scientifiques de niveau recherche, publiés ou non, émanant des établissements d'enseignement et de recherche français ou étrangers, des laboratoires publics ou privés. 


\title{
Investigation of elastic modes propagating in multi-wire helical waveguides
}

\author{
Fabien Treyssède ${ }^{*, a}$, Laurent Laguerre ${ }^{\mathrm{a}}$ \\ ${ }^{a}$ Laboratoire Central des Ponts et Chaussées, BP 4129, 44341 Bouguenais, France
}

\begin{abstract}
Elastic guided waves have some potential for non-destructive inspection of civil engineering multi-wire steel cables. However, wave propagation inside such structures is not yet fully understood. This paper investigates multi-wire helical waveguides with special attention to the common seven-wire strand configuration (one straight core surrounded by one layer of six helical wires). A helical coordinate system is first proposed. Though non-orthogonal, this system preserves translational invariance along the helix centreline to explicitly perform a spatial Fourier transform. Then, it is shown that for the analysis of multi-wire helical strands a twisting system - which is a special case of helical systems - is translationally invariant. A semi-analytical finite element method is developed reducing the problem on the cross-section only. A straightforward computation of energy velocity is proposed. Dispersion curves for a single straight wire and a helical wire are first computed to verify the adequacy of the twisting system. Finally the seven-wire strand is analysed using simplified contact conditions. Theoretical dispersion curves are compared to low-frequency magnetostrictive measurements. Good agree-
\end{abstract}

\footnotetext{
${ }^{*}$ Corresponding author

Email address: fabien.treyssede@lcpc.fr (Fabien Treyssède)
} 
ment is found for the first compressional-like mode and its associated veering central frequency ('notch frequency').

Key words: waveguide, propagation, helical, strand, finite element, experiment

\section{Introduction}

Elastic guided waves are attractive tools for non-destructive testing and structural health monitoring because of their potential ability to inspect, in a single measurement, the whole width of the structure over an appreciable length along the guiding direction. Theoretical modelling effort is generally required to enhance physical insight into the complex behaviour of guided waves, which are multimodal and dispersive. Though cylindrical elastic waveguides have extensively been studied since the early works of Pochhammer in 1876 and Chree in 1889 [1, 2], less attention has been paid to helical and multi-wire waveguides. A typical application in civil engineering concerns steel multi-wire cables, which are widely employed in bridges (suspended, cable-stayed and prestressed) and anchored retaining wall constructions. These civil engineering structures suffer from ageing and degradation due to corrosion and fatigue.

The basic element of these cables is usually a seven-wire strand, consisting of one straight cylindrical core wire surrounded by one layer of six helical wires. The simplest and usual way to consider wave propagation in a sevenwire strand is to approximate the latter by an infinite cylinder of equivalent diameter. Various experimental studies have been conducted in the literature for the cylindrical bar and the seven wire strand, trying to find similarities 
between both behaviours that could be interpreted from Pochhammer-Chree solutions, either for the bare waveguide case $[3,4,5,6,7]$ or the embedded one $[8,9]$. Even if the non-destructive inspection strategy of a seven-wire strand with guided ultrasonic waves has gained from this approach, the authors point out the limits of Pochhammer-Chree solutions for the accurate interpretation of experimental data. Indeed, the theoretical understanding of guided ultrasonic waves in multi-wire strands is still challenging because of the complexity of this structure, due to the helical geometry of peripheral wires, the inter-wire coupling and contact effects, the presence of applied loads and concrete embedment (if any).

The aim of this paper is to propose a numerical method to study elastic wave propagation along multi-wire helical waveguides in order to help the interpretation of guided waves in these structures. According to the complexity of the problem, a computational approach is preferred to a purely mathematical approach. As a first step, inter-wire contact conditions will be simplified, and prestress and embedment will not be considered.

In order to deal with complex geometries, some of the most popular and efficient numerical techniques involve finite element (FE) methods. The socalled semi-analytical finite element (SAFE) method is a first popular approach to study uniform straight waveguides of arbitrary cross-section - see for instance Gavric [10], Hayashi et al. [11], Damljanovic and Weaver [12], Bartoli et al. [13]. This method assumes an axial dependence of the form $e^{i k s}$ ( $k$ and $s$ are the wavenumber and distance along the waveguide axis respectively), hence reducing the problem from three to two dimensions (only the cross-section is meshed). The SAFE method has also been extended to 
curved waveguides. Demma et al. [14], Finnveden and Fraggstedt [15] investigated toroidal waveguides. Onipede and Dong [16] extended SAFE methods to study uniformly pretwisted waveguides along a straight axis.

A second approach is based on the theory of wave propagation in periodic structures from Floquet's principle (this approach is more general because non-uniform waveguides can be analysed). A review can be found in Ref. [17]. Based on a general theory presented by Mead [18], some periodic FE approaches and procedures have then been developed - see for instance Gry and Gontier [19], Mace et al. [20]. The periodic FE method allows to study the single repetitive substructure alone, thanks to the application of a set of periodic boundary conditions involving a propagation constant corresponding to the eigenvalue. Periodic FE methods only need the mesh of one repetitive cell.

Recently, Treyssède extended both periodic FE [21] and SAFE [22] methods to model a single helical wire. Both methods are based on a specific curvilinear coordinate system attached to the helical waveguide. Such a system is non orthogonal but remains translationally invariant along the helix centreline, which allows a Fourier analysis (or a Floquet analysis with an arbitrarily small repetitive cell). In this paper, it is shown how the proposed approach can also be readily used to study a multi-wire helical waveguide. In Sec. 2, the helical coordinate system is first recalled. A particular twisting coordinate system is then discussed for the analysis of multi-wire waveguides. A SAFE method, whose weak variational formulation is rewritten in terms of the helical coordinate system, is then presented in Sec. 3. Section 4 gives preliminary results for a single wire both for the cylindrical and 
helical cases. Section 5 shows some numerical results (including dispersion curves and modeshapes) for the seven-wire strand configuration in civil engineering. From longitudinally-polarized magnetostrictive measurements in the low-frequency range $(<500 \mathrm{kHz})$, a first experimental validation of theoretical dispersion curves is performed both for the single constitutive wires (cylindrical and helical) as well as the assembled seven-wire strand.

\section{Translationally invariant coordinate systems}

Let $(x, y, s)$ denote a curvilinear coordinate system attached to a curved waveguide, where $x$ and $y$ are the cross-section coordinates and $s$ is the axial coordinate. In order to still speak of propagation modes, the physical system must be translationally invariant. In other words, one has to assume that an exponential axial dependence $e^{i k s}$ can be separated from all field components (which is equivalent to perform a spatial Fourier analysis in the $s$ direction). This assumption indeed requires to meet the following three conditions:

1. the cross-section of the waveguide does not vary along $s$;

2. the material properties do not vary along $s$;

3. the coordinate system $(x, y, s)$ is such that $s$ does not appear explicitly in the coefficients of the equilibrium equations.

The first condition is an obvious geometrical condition. Condition 2 will be assumed to be satisfied throughout this work. The aim of this section is to propose some coordinate systems verifying the third non-trivial condition. For a fundamental introduction to the use of general curvilinear coordinate systems, the reader may refer to Chapter 2 of Ref. [23] for instance. 


\subsection{Helical coordinate system}

A helical coordinate system is now built. One starts by defining the helix centreline curve, described by the following position vector in the Cartesian orthonormal basis $\left(\mathbf{e}_{X}, \mathbf{e}_{Y}, \mathbf{e}_{Z}\right)$ :

$$
\mathbf{R}(s)=R \cos \left(\frac{2 \pi}{l} s+\theta\right) \mathbf{e}_{X}+R \sin \left(\frac{2 \pi}{l} s+\theta\right) \mathbf{e}_{Y}+\frac{L}{l} s \mathbf{e}_{Z}
$$

where $l=\sqrt{L^{2}+4 \pi^{2} R^{2}}$ is the curvilinear length of one helix step. $R$ and $L$ are respectively the radius of the centreline in the $(X, Y)$ Cartesian plane and the helix step along the $Z$ axis (see Fig. 1). $\theta$ is the helix phase angle in the $Z=0$ plane. For instance, a seven-wire strand comprises six helical waveguides with $\theta=(k-1) \pi / 3, k=1, \ldots, 6$. The unit tangent, normal and binormal vectors to the centreline are respectively obtained from $\mathbf{T}=d \mathbf{R} / d s$ and the Serret-Frenet formulae [24]: $d \mathbf{T} / d s=-\kappa \mathbf{N}, d \mathbf{N} / d s=\tau \mathbf{B}+\kappa \mathbf{T}$ and $d \mathbf{B} / d s=-\tau \mathbf{N}(\mathbf{B}=\mathbf{T} \wedge \mathbf{N})$. Note that $\mathbf{N}$ is oriented outward the curvature in this paper (this is just a matter of taste). For a helix, both the curvature $\kappa=4 \pi^{2} R / l^{2}$ and torsion $\tau=2 \pi L / l^{2}$ are constant. In the Cartesian basis, $\mathbf{N}, \mathbf{B}$ and $\mathbf{T}$ are expressed as:

$$
\begin{gathered}
\mathbf{N}(s)=\cos \left(\frac{2 \pi}{l} s+\theta\right) \mathbf{e}_{X}+\sin \left(\frac{2 \pi}{l} s+\theta\right) \mathbf{e}_{Y} \\
\mathbf{B}(s)=-\frac{L}{l} \sin \left(\frac{2 \pi}{l} s+\theta\right) \mathbf{e}_{X}+\frac{L}{l} \cos \left(\frac{2 \pi}{l} s+\theta\right) \mathbf{e}_{Y}-\frac{2 \pi R}{l} \mathbf{e}_{Z}, \\
\mathbf{T}(s)=-\frac{2 \pi R}{l} \sin \left(\frac{2 \pi}{l} s+\theta\right) \mathbf{e}_{X}+\frac{2 \pi R}{l} \cos \left(\frac{2 \pi}{l} s+\theta\right) \mathbf{e}_{Y}+\frac{L}{l} \mathbf{e}_{Z}
\end{gathered}
$$

In this way, a new coordinate system can be built from the orthonormal basis $(\mathbf{N}, \mathbf{B}, \mathbf{T})$, for which any position vector $\mathbf{\Phi}=X \mathbf{e}_{X}+Y \mathbf{e}_{Y}+Z \mathbf{e}_{Z}$ is expressed as follows:

$$
\boldsymbol{\Phi}(x, y, s)=\mathbf{R}(s)+x \mathbf{N}(s)+y \mathbf{B}(s)
$$




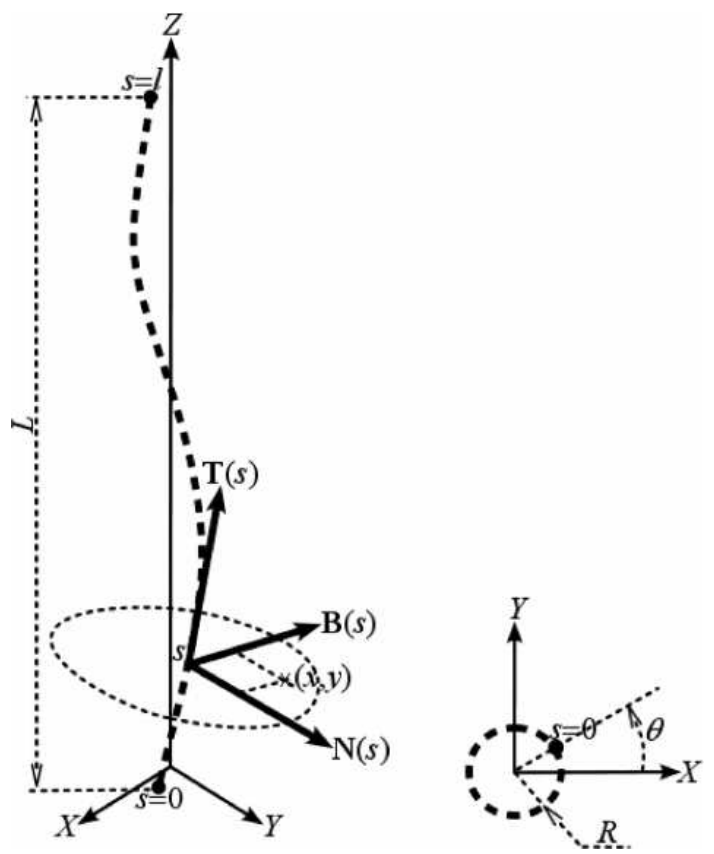

Figure 1: Left: helix centreline curve (one step) with its associated Serret-Frenet basis and $(x, y, s)$ helical coordinate system. Right: plane view. The point $s=0$ lies in the $(X, Y)$ plane. 
yielding the non-orthogonal covariant basis $(\partial \Phi / \partial x, \partial \boldsymbol{\Phi} / \partial y, \partial \Phi / \partial s)$ denoted by $\left(\mathbf{g}_{1}, \mathbf{g}_{2}, \mathbf{g}_{3}\right)$ :

$$
\begin{gathered}
\mathbf{g}_{1}=\mathbf{N}(s), \mathbf{g}_{2}=\mathbf{B}(s) \\
\mathbf{g}_{3}=-\tau y \mathbf{N}(s)+\tau x \mathbf{B}(s)+(1+\kappa x) \mathbf{T}(s) .
\end{gathered}
$$

The covariant metric tensor, defined by $(\mathbf{g})_{i j}=\mathbf{g}_{i} \cdot \mathbf{g}_{j}$ is:

$$
\mathbf{g}=\left[\begin{array}{ccc}
1 & 0 & -\tau y \\
0 & 1 & \tau x \\
-\tau y & \tau x & \tau^{2}\left(x^{2}+y^{2}\right)+(1+\kappa x)^{2}
\end{array}\right]
$$

g does not depend on $s$. As a consequence, the coefficients of any partial differential operators expressed in the so-defined coordinate system are independent on $s$ too (condition 3 is hence satisfied).

For clarity the contravariant basis $\left(\mathbf{g}^{1}, \mathbf{g}^{2}, \mathbf{g}^{3}\right)$, defined by $\mathbf{g}^{i} \cdot \mathbf{g}_{j}=\delta_{j}^{i}$, is given by:

$$
\begin{gathered}
\mathbf{g}^{1}=\mathbf{N}(s)+\frac{\tau y}{1+\kappa x} \mathbf{T}(s), \\
\mathbf{g}^{2}=\mathbf{B}(s)-\frac{\tau x}{1+\kappa x} \mathbf{T}(s), \mathbf{g}^{3}=\frac{1}{1+\kappa x} \mathbf{T}(s)
\end{gathered}
$$

yielding the following contravariant metric tensor, defined by $g^{m n}=\mathbf{g}^{m} \cdot \mathbf{g}^{n}$ :

$$
\mathbf{G}=\mathbf{g}^{-1}=\frac{1}{g}\left[\begin{array}{ccc}
g+(\tau y)^{2} & -\tau^{2} x y & \tau y \\
-\tau^{2} x y & g+(\tau x)^{2} & -\tau x \\
\tau y & -\tau x & 1
\end{array}\right]
$$

where $g=(1+\kappa x)^{2}$ is the determinant of $\mathbf{g}$. The Christoffel symbol of the second kind $\Gamma_{i j}^{k}$, defined by $\Gamma_{i j}^{k}=\mathbf{g}_{i, j} \cdot \mathbf{g}^{k}$, can be readily calculated from the 
Serret-Frenet formulae:

$$
\begin{gathered}
\Gamma_{11}^{k}=\Gamma_{12}^{k}=\Gamma_{21}^{k}=\Gamma_{22}^{k}=0, \Gamma_{23}^{2}=\Gamma_{32}^{2}=\Gamma_{23}^{3}=\Gamma_{32}^{3}=0, \\
\Gamma_{33}^{1}=\frac{-\kappa \tau y^{2}}{1+\kappa x}-\kappa(1+\kappa x)-\tau^{2} x, \Gamma_{33}^{2}=\frac{\kappa \tau^{2} x y}{1+\kappa x}-\tau^{2} y, \Gamma_{33}^{3}=\frac{-\kappa \tau y}{1+\kappa x}, \\
\Gamma_{13}^{1}=\Gamma_{31}^{1}=\frac{\kappa \tau y}{1+\kappa x}, \Gamma_{23}^{1}=\Gamma_{32}^{1}=-\tau, \Gamma_{13}^{2}=\Gamma_{31}^{2}=\frac{-\kappa \tau x}{1+\kappa x}+\tau, \Gamma_{13}^{3}=\Gamma_{31}^{3}=\frac{\kappa}{1+\kappa x} .
\end{gathered}
$$

\subsection{Particular system for a multi-wire waveguide}

Consider wires of circular cross-section in the normal plane to the guiding axis. The cross-section of a single helical wire does not change along the helical axis $s$ (condition 1 is also fulfilled). Hence for helical waveguides, the previously described coordinate system allows a wave mode analysis. The analysis of a single straight wire, corresponding to the special case $\kappa=\tau=0$, is also readily performed. However, a question arises about the choice of the invariant coordinate system to be used for a multi-wire helical waveguide, made of both straight and helical wires. Of course, the choice $\kappa=\tau=0$ cannot be applied because condition 1 would not be satisfied for helical wires, and similarly $\kappa \neq 0, \tau \neq 0$ cannot be applied because it would not be satisfied for straight wires. The same problem also occurs for any additional layer of peripheral wires because each layer would have a different curvature and torsion.

The adequate system is indeed given by $\kappa=0$ and $\tau=2 \pi / L$. It corresponds to a twisting coordinate system along the $Z$ axis $(s \equiv Z)$ with axial periodicity $L$. The $(x, y)$ plane rotates around $Z$ but remains parallel to $(X, Y)$. With this choice, a central straight wire (cylinder) has an invariant circular cross-section along $Z$ ("a twisted cylinder remains a cylinder"). Furthermore, the cross-sections of peripheral helical wires do not change ei- 
ther along $Z$. In Sec. 4, it will be checked that this coordinate system yields right results for both a cylindrical and a helical waveguides. It should also be noted that this system coincides with the one proposed in Ref. [16] for the analysis of pretwisted waveguides. It also had already been considered in electromagnetics $[25,26]$.

However, one has to be careful with such a system because the crosssection shape of helical wires is no longer circular (the cutting plane being not normal to the helix centreline). Let us find the cross-section cut by the plane $Z=0$. Equation (3) yields:

$$
\left\{\begin{array}{l}
X=(R+x) \cos \left(\frac{2 \pi}{l} s+\theta\right)-y \frac{L}{l} \sin \left(\frac{2 \pi}{l} s+\theta\right) \\
Y=(R+x) \sin \left(\frac{2 \pi}{l} s+\theta\right)+y \frac{L}{l} \cos \left(\frac{2 \pi}{l} s+\theta\right) \\
Z=\frac{L}{l} s-\frac{2 \pi R}{l} y
\end{array}\right.
$$

so that $s=2 \pi y R / L$ at $Z=0$. In a helical coordinate system, the surface of a helical wire with a circular cross-section of radius $a$ is given by $(x, y)=$ $(a \cos t, a \sin t)$, with $t \in[0 ; 2 \pi]$ and $s \in \mathbb{R}$. Consequently, the cross-section shape can be parametrized as follows:

$$
\left\{\begin{array}{l}
X(t)=(R+a \cos t) \cos (\alpha a \sin t+\theta)-a \frac{L}{l} \sin t \sin (\alpha a \sin t+\theta) \\
Y(t)=(R+a \cos t) \sin (\alpha a \sin t+\theta)+a \frac{L}{l} \sin t \cos (\alpha a \sin t+\theta)
\end{array}\right.
$$

where $\alpha=4 \pi^{2} R /(l L)$. With a twisting coordinate system, this shape must be used for generating the FE mesh (see Sec. 4).

As a side remark, the particular case $\tau=0$ degenerates into a coordinate system attached to a toroidal waveguide of curvature $\kappa$ (of no interest in this paper). 


\section{Numerical method}

\subsection{SAFE formulation}

One assumes a linearly elastic material, small strains and displacements with a time harmonic $e^{-i \omega t}$ dependence. There is no external force for the purpose of studying propagation modes. The $3 \mathrm{D}$ variational formulation governing elastodynamics is given by:

$$
\int_{\Omega} \delta \boldsymbol{\epsilon}^{T} \boldsymbol{\sigma} d V-\omega^{2} \int_{\Omega} \rho \delta \mathbf{u}^{T} \mathbf{u} d V=0
$$

for any kinematically admissible trial displacement field $\delta \mathbf{u}=\left[\begin{array}{lll}\delta u_{n} & \delta u_{b} & \delta u_{t}\end{array}\right]^{T}$. Components in the orthonormal Serret-Frenet basis $(\mathbf{N}, \mathbf{B}, \mathbf{T})$ are preferred here. $\delta \boldsymbol{\epsilon}$ denotes the virtual strain vector $\left[\delta \epsilon_{n n} \delta \epsilon_{b b} \delta \epsilon_{t t} 2 \delta \epsilon_{n b} 2 \delta \epsilon_{n t} 2 \delta \epsilon_{b t}\right]^{T}$ and $\boldsymbol{\sigma}$ is the stress vector $\left[\sigma_{n n} \sigma_{b b} \sigma_{t t} \sigma_{n b} \sigma_{n t} \sigma_{b t}\right]^{T}$. The superscript $T$ denotes the matrix transpose. $\rho$ is the material density and $\Omega$ is the structural volume. The stress-strain relationship is $\boldsymbol{\sigma}=\mathbf{C} \boldsymbol{\epsilon}$, where $\mathbf{C}$ is the matrix of material properties (whose components are also given with respect to the Serret-Frenet basis). The volume element $d V$ is given by $d V=\sqrt{g} d x d y d s$, where $g$ is the determinant of the metric tensor previously defined. $\sqrt{g}$ can be understood as the Jacobian of the transformation.

The strain-displacement relation can be written as follows:

$$
\boldsymbol{\epsilon}=\left(\mathbf{L}_{x y}+\mathbf{L}_{s} \partial / \partial s\right) \mathbf{u}
$$

where $\mathbf{L}_{x y}$ is the operator containing all terms except the derivatives with respect to the $s$-axis. Now an exponential $e^{i k s}$ is assumed for $\mathbf{u}\left(e^{-i k s}\right.$ for $\delta \mathbf{u})$ and separated from all field components, $k$ being the axial wavenumber. $\partial / \partial s$ can be replaced by $\pm i k$. The problem is hence reduced from three 
to two dimensions (from the volume $\Omega$ to the cross-section $S$ of the waveguide). Then, the FE discretization of Eq. (11) finally leads to the following eigenvalue problem for the column vector $\mathbf{U}$ containing nodal displacements:

$$
\left\{\mathbf{K}_{1}-\omega^{2} \mathbf{M}+i k\left(\mathbf{K}_{2}-\mathbf{K}_{2}^{T}\right)+k^{2} \mathbf{K}_{3}\right\} \mathbf{U}=\mathbf{0}
$$

with the following elementary matrices:

$$
\begin{aligned}
& \mathbf{K}_{1}^{e}=\int_{S^{e}} \mathbf{N}^{e T} \mathbf{L}_{x y}^{T} \mathbf{C L}_{x y} \mathbf{N}^{e} \sqrt{g} d S, \mathbf{K}_{2}^{e}=\int_{S^{e}} \mathbf{N}^{e T} \mathbf{L}_{x y}^{T} \mathbf{C L}_{s} \mathbf{N}^{e} \sqrt{g} d S, \\
& \mathbf{K}_{3}^{e}=\int_{S^{e}} \mathbf{N}^{e T} \mathbf{L}_{s}^{T} \mathbf{C L}_{s} \mathbf{N}^{e} \sqrt{g} d S, \mathbf{M}^{e}=\int_{S^{e}} \rho \mathbf{N}^{e T} \mathbf{N}^{e} \sqrt{g} d S
\end{aligned}
$$

where $\mathbf{N}^{e}$ is a matrix of nodal interpolating functions of displacement on the element and $d S=d x d y$.

The solution of Eq. (13) yields the propagation modes. Because of the symmetry of $\mathbf{K}_{1}, \mathbf{K}_{3}$ and $\mathbf{M}$ and using the property $\operatorname{det} \mathbf{A}^{T}=\operatorname{det} \mathbf{A}(\mathbf{A}$ is any matrix), it can easily be checked that if $k$ is an eigenvalue of (13), then $-k$ is also an eigenvalue. Hence, the eigenproblem has two sets of eigensolutions $\left(k_{j}, \mathbf{U}_{j}^{+}\right)$and $\left(-k_{j}, \mathbf{U}_{j}^{-}\right)(j=1, \ldots, n)$, representing $n$ positivegoing and $n$ negative-going wave types ( $n$ being the number of degrees of freedom (dofs)). With no damping, eigensolutions for which $k_{j}$ is purely real, purely imaginary and fully complex represent propagating waves, evanescent waves and inhomogeneous waves (decaying but oscillatory) respectively.

At fixed real $k$, the eigenproblem (13) is linear for finding $\omega^{2}$. This simpler approach is useful only in the case of propagating modes in undamped systems. Given $\omega$ and finding $k$, the eigenproblem is quadratic. It can be recast into a generalized linear eigensystem written for $\left[\mathbf{U}^{T} k \mathbf{U}^{T}\right]^{T}$ in order to be solved by standard numerical solvers - see Treyssède [22] for instance or Tisseur and Meerbergen [27] for more details. 


\subsection{Expression of operators in curvilinear coordinate system}

The strain-displacement relationship $\boldsymbol{\epsilon}=1 / 2\left(\nabla \mathbf{u}+\nabla \mathbf{u}^{T}\right)$ (here, $\boldsymbol{\epsilon}$ must be understood as a second order tensor), must be written in a general nonorthogonal curvilinear coordinate system. Using product and differentiation rules of such a system (see for instance Chapter 2 of Ref. [23]), it can be shown that the strain tensor is given by:

$$
\epsilon_{i j}=1 / 2\left(u_{i, j}+u_{j, i}\right)-\Gamma_{i j}^{k} u_{k}
$$

Subscripts $i=1,2,3$ denote covariant components with respect to the contravariant basis $\left(\mathbf{g}^{1}, \mathbf{g}^{2}, \mathbf{g}^{3}\right)$. The notation $(\cdot)_{, i}(i=1,2,3)$ is used for derivatives with respect to $x, y$ and $s$ respectively.

Note that covariant components generally have non-physical units and the contravariant basis is non-orthogonal. Physical components with respect to the orthonormal Serret-Frenet basis $(\mathbf{N}, \mathbf{B}, \mathbf{T})$ are preferred. Then we have the following transformations:

$$
u_{i}=J_{\alpha i} u_{\alpha}, \epsilon_{i j}=J_{\alpha i} \epsilon_{\alpha \beta} J_{\beta j}
$$

with:

$$
\mathbf{J}=\left[\begin{array}{ccc}
1 & 0 & -\tau y \\
0 & 1 & \tau x \\
0 & 0 & 1+\kappa x
\end{array}\right]
$$

where the greek subscripts $\alpha=n, b, t$ denote components with respect to $(\mathbf{N}, \mathbf{B}, \mathbf{T})$. Note that $\mathbf{g}=\mathbf{J}^{T} \mathbf{J}$.

Finally from Eqs. (15)-(17) and after calculations, the following operators 
$\mathbf{L}_{x y}$ and $\mathbf{L}_{s}$ involved in Eq. (12) are:

$$
\mathbf{L}_{x y}=\frac{1}{1+\kappa x}\left[\begin{array}{ccc}
(1+\kappa x) \partial / \partial x & 0 & 0 \\
0 & (1+\kappa x) \partial / \partial y & 0 \\
\kappa & 0 & \tau y \partial / \partial x-\tau x \partial / \partial y \\
(1+\kappa x) \partial / \partial y & (1+\kappa x) \partial / \partial x & 0 \\
\tau y \partial / \partial x-\tau x \partial / \partial y & -\tau & -\kappa+(1+\kappa x) \partial / \partial x \\
\tau & \tau y \partial / \partial x-\tau x \partial / \partial y & (1+\kappa x) \partial / \partial y
\end{array}\right]
$$

and

$$
\mathbf{L}_{s}=\frac{1}{1+\kappa x}\left[\begin{array}{lll}
0 & 0 & 0 \\
0 & 0 & 0 \\
0 & 0 & 1 \\
0 & 0 & 0 \\
1 & 0 & 0 \\
0 & 1 & 0
\end{array}\right]
$$

The components of $\mathbf{C}$ must also be expressed with respect to the SerretFrenet basis. For an isotropic material, one has:

$$
\mathbf{C}=\frac{E}{(1+\nu)(1-2 \nu)}\left[\begin{array}{cccccc}
1-\nu & \nu & \nu & 0 & 0 & 0 \\
\nu & 1-\nu & \nu & 0 & 0 & 0 \\
\nu & \nu & 1-\nu & 0 & 0 & 0 \\
0 & 0 & 0 & (1-2 \nu) / 2 & 0 & 0 \\
0 & 0 & 0 & 0 & (1-2 \nu) / 2 & 0 \\
0 & 0 & 0 & 0 & 0 & (1-2 \nu) / 2
\end{array}\right]
$$

where $E$ is the Young modulus and $\nu$ denotes the Poisson coefficient. 


\subsection{Computation of energy velocity}

The energy velocity is an important wave property that remains appropriate even for damped media [28] (as opposed to the group velocity definition $v_{g}=\partial \omega / \partial k$, which is not generally valid for damped waveguides). From the previously defined SAFE matrices, a straightforward computation of energy velocity is proposed in this subsection.

The cross-section and time averaged energy velocity in waveguides is defined as follows [29]:

$$
v_{e}=\frac{\int_{S} \overline{\mathbf{P}} \cdot \mathbf{n} d S}{\int_{S}\left(\bar{E}_{k}+\bar{E}_{p}\right) d S}
$$

where bars denote time averaging. $\mathbf{n}$ is the unit vector along the propagation direction (normal to the cross-section). $\mathbf{P}$ is the Poynting vector, $E_{k}$ and $E_{p}$ are the kinetic and potential energies, defined by:

$$
P_{\alpha}=-\sigma_{\alpha \beta} \dot{u}_{\beta}, E_{k}=\frac{1}{2} \rho \dot{u}_{\alpha} \dot{u}_{\alpha}, E_{p}=\frac{1}{2} \epsilon_{\alpha \beta} \sigma_{\alpha \beta} .
$$

Once the eigensystem (13) is solved, the energy velocity of any eigenmode $(k, \mathbf{U})$ can indeed be directly post-processed from SAFE matrices. First, it can easily be deduced that:

$$
\begin{gathered}
\int_{S} \bar{E}_{k} d S=\frac{\omega^{2}}{4} \operatorname{Re}\left(\mathbf{U}^{T *} \mathbf{M} \mathbf{U}\right), \\
\int_{S} \bar{E}_{p} d S=\frac{1}{4} \operatorname{Re}\left\{\mathbf{U}^{T *}\left(\mathbf{K}_{1}+i k\left(\mathbf{K}_{2}-\mathbf{K}_{2}^{T}\right)+k^{2} \mathbf{K}_{3}\right) \mathbf{U}\right\} .
\end{gathered}
$$

The computation of the cross-section and time averaged Poynting vector requires further developments but can also be simply expressed. Noticing that $\mathbf{n}=\mathbf{T}$, one has:

$$
\overline{\mathbf{P}} \cdot \mathbf{n} d S=\frac{\omega}{2} \operatorname{Im}\left\{u_{\alpha}^{*} \sigma_{\alpha t} d S\right\}
$$


Then, it can be checked that $u_{\alpha}^{*} \sigma_{\alpha t}=\mathbf{u}^{T *} \mathbf{L}_{s}^{T} \boldsymbol{\sigma}(1+\kappa x)=\mathbf{u}^{T *} \mathbf{L}_{s}^{T} \mathbf{C}\left(\mathbf{L}_{x y}+\right.$ $\left.i k \mathbf{L}_{s}\right) \mathbf{u}(1+\kappa x)$, so that the following useful expression holds for the averaged Poynting vector:

$$
\int_{S} \overline{\mathbf{P}} \cdot \mathbf{n} d S=\frac{\omega}{2} \operatorname{Im}\left\{\mathbf{U}^{T *}\left(\mathbf{K}_{2}^{T}+i k \mathbf{K}_{3}\right) \mathbf{U}\right\}
$$

Expressions (23) and (25) allow a direct computation of the energy velocity defined by Eq. (21).

\section{Preliminary results}

The aim of this section is to verify that a twisting system yields the same physical results as those obtained with a straight coordinate system for a cylindrical waveguide, and similarly as those obtained with a helical system for a helical waveguide. The material is assumed to be isotropic, with no material damping. A value of 0.30 is chosen for the Poisson coefficient. One considers a waveguide with a circular cross-section of radius $a$. The normalized frequency is given by $\omega a / c_{s}, c_{s}=\sqrt{E / 2 \rho(1+\nu)}$ denoting the shear wave velocity. FE computations are held at fixed real wavenumbers $k$. Six-node triangles meshes are used. The helix lay angle $\phi$ is defined from: $\tan \phi=2 \pi R / L$.

\subsection{Cylindrical waveguide}

For a cylindrical waveguide, we have $\phi=0$ and its axis corresponds to the Cartesian $Z$ axis. As previously mentioned, the Cartesian system $(X, Y, Z)$, which is the special case $\kappa=\tau=0$, obviously yields a translational invariance. As clearly shown in this subheading, a cylinder can also be analysed with a twisting coordinate system $(\kappa=0, \tau \neq 0)$, where $\tau$ can be any value. 

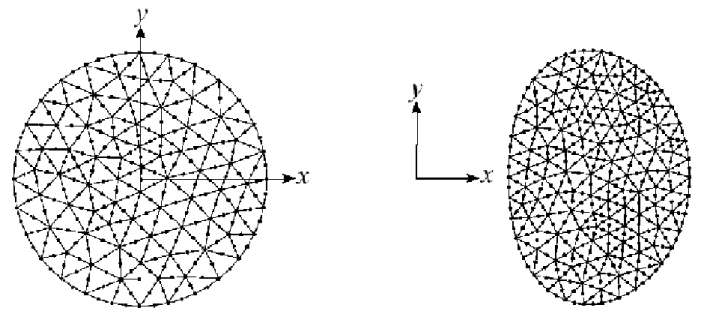

Figure 2: Left: cross-section FE mesh of a cylindrical waveguide (and of a helical waveguide analysed with the helical coordinate system). Right: cross-section FE mesh of a helical waveguide $R=2 a, \phi=\pi / 4$ analysed with the twisting coordinate system.

Both cases $(\kappa=0, \tau=0)$ (reference solution) and $(\kappa=0, \tau \neq 0)$ are computed. In order to check the adequacy of the twisting system, we choose a high torsion $\tau a=0.5$ (high rotation rate). For clarity, Fig. 2 (left) illustrates the cross-section FE mesh used (the same for both coordinate systems). Figure 3 exhibits the dispersion curves (normalized frequency vs. wavenumber) obtained from both systems. Wavenumbers of compressional $L(0, n)$ and torsional $T(0, n)$ modes are left unchanged because of their axisymmetry.

However, one must be careful when interpreting the strong differences found for wavenumbers of flexural modes $F(m, n)$, which are non-axisymmetric. With a Cartesian system, the wavenumbers of $F(m, n)$ modes occur in pairs of double roots. With a twisting system, their phase velocity become distinct due to the rotation of the $(x, y)$ plane around $Z$ (the axisymmetry is broken), their wavenumbers being translated by $\pm m \tau a$ (see Fig. 4 for clarity). This was also obtained in Ref. [16], where further explanations can be found.

Note that a wavenumber translation from $k a$ to $k a \pm m \tau a$ does not affect the slopes of curves in Fig. 3. Hence, the group velocity $v_{g}=\partial \omega / \partial k$ should remain unchanged with the twisting system. Because no damping is consid- 


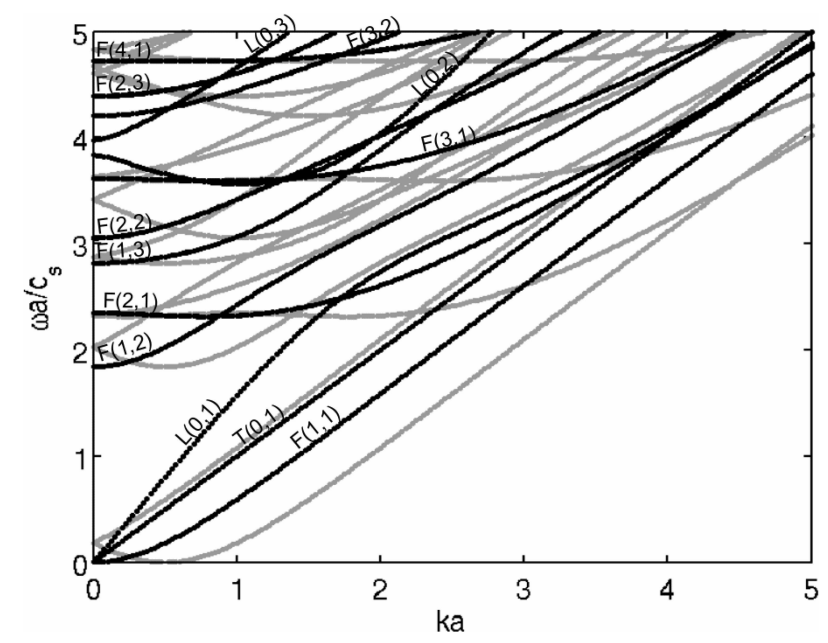

Figure 3: Plot of normalized frequency vs. wavenumber for a cylindrical waveguide. Black: Cartesian system (reference), gray: twisting system.

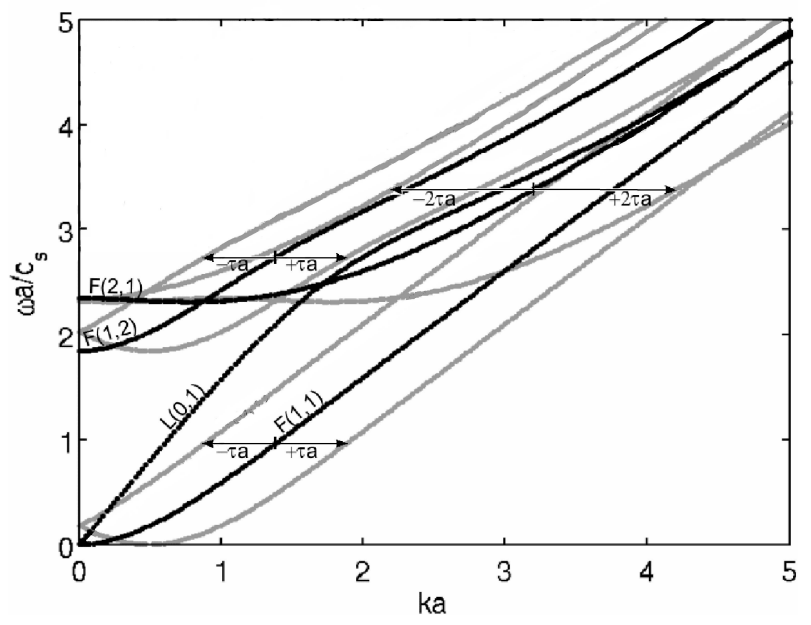

Figure 4: Wavenumber translation for some examples of $F(m, n)$ modes (same legend as Fig. 3). The wavenumber of the $L(0,1)$ mode is left unchanged (axisymmetric mode). 


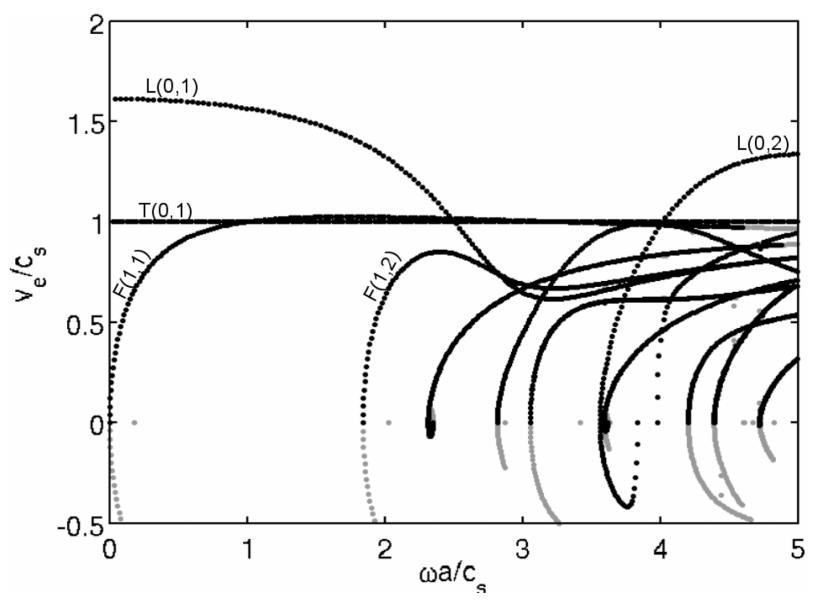

Figure 5: Plot of normalized frequency vs. wavenumber for a cylindrical waveguide. Black: Cartesian system (reference), gray: twisting system.

ered here, the group velocity is also equal to the energy velocity [28], and can be computed from Eqs. (21), (23) and (25). The energy velocity is plotted in Fig. 5: the same curves are obtained for the straight system and the twisting system. Consequently, both systems clearly yield equivalent results from a physical point of view.

\subsection{Helical waveguide}

One considers a waveguide with a helix radius $R=2 a$ and a strong helix lay angle $\phi=\pi / 4$. This yields $(\kappa a=0.25, \tau a=0.25)$ for the helical system and $(\kappa a=0, \tau a=0.5)$ for the twisting system. The computed solution given by the helical system, for which the cross-section is circular (same mesh as Fig. 2 - left), is considered as the reference solution (see Refs. [21, 22]). Figure 2 (right) exhibits the cross-section mesh associated with the twisting system. This cross-section, corresponding to a $Z=0$ plane 


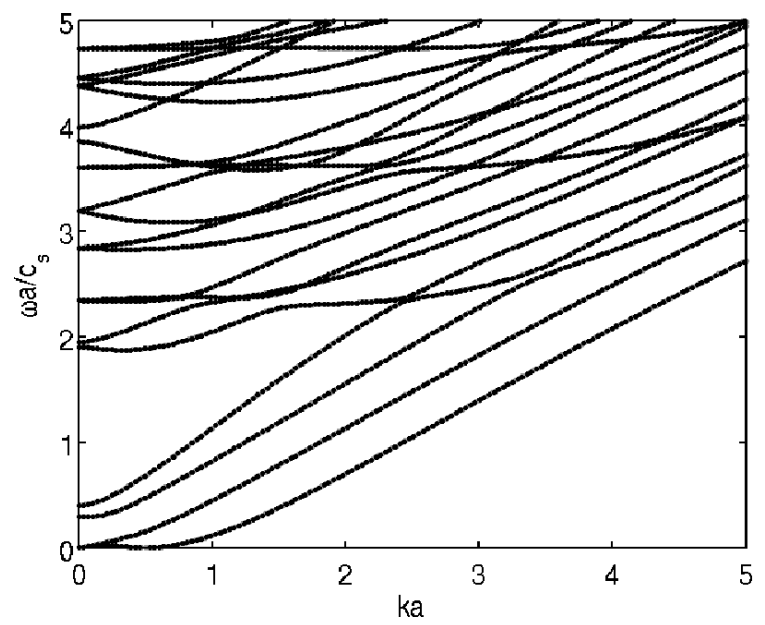

Figure 6: Plot of normalized frequency vs. wavenumber for the helical waveguide $R=2 a$, $\phi=\pi / 4$. Black: helical system (reference), gray: twisting system.

cut as parametrized by Eq. (10), is no longer circular as considered earlier. One must also be careful when considering results obtained with the helical system: in order to transform results from a curvilinear helical $s$-axis to a straight $Z$-axis, the energy velocities must be divided by the step ratio $l / L$ (the wavenumbers $k$ must be multiplied by $l / L$ ). Figure 6 shows the dispersion curves computed for both the helical and twisting coordinate systems. No difference is observed in wavenumber predictions, demonstrating the adequacy of the twisting system. Note that the differences previously observed for flexural modes inside a cylinder do not occur here because both systems rotate at the same rate along $Z$. Obviously, results for energy velocities are also identical. 


\section{Analysis of the seven-wire strand}

Now wave modes propagating inside a typical seven-wire steel strand are studied both numerically and experimentally. The core wire radius is $a=2.7 \mathrm{~mm}$. The helical wires have a radius equal to $0.967 a$ and a pitch $L=240 \mathrm{~mm}$, yielding a lay angle of $\phi=7.9^{\circ}$. Mechanical properties are as follows: $E=2.17 \mathrm{e} 11 \mathrm{~Pa}, \nu=0.28, \rho=7800 \mathrm{~kg} / \mathrm{m}^{3}$. It is outlined that peripheral wires do not contact each other (this is a rather widespread design criterion for minimizing friction effects). As before, FE computations are performed at fixed real wavenumbers $k$ and six-node triangles meshes are used. The energy velocity defined by Eq. (21) is computed thanks to the useful formula (23) and (25).

\subsection{Numerical results}

The twisting coordinate system is $(\kappa a=0, \tau a=0.0705)$. The FE mesh, corresponding to a $Z=0$ plane cut, is given by Fig. 7. Though not visible in the figure, there is no physical contact between peripheral helical wires and their cross-section is not exactly circular (even if this non-circularity has a negligible effect here). For a better accuracy of numerical results, the mesh has been strongly refined at physical contact points, yielding 6975 dofs. For simplicity, one assumes stick contact conditions between the core and outside wires (no slip, no separation and no friction are considered). This is equivalent to suppose that friction is high enough to prevent any slipping between wires, and to consider continuous displacement in every directions at the six contact points. The results are computed at fixed real wavenumbers $k$ for the normalized frequency range $[0 ; 2]$. 


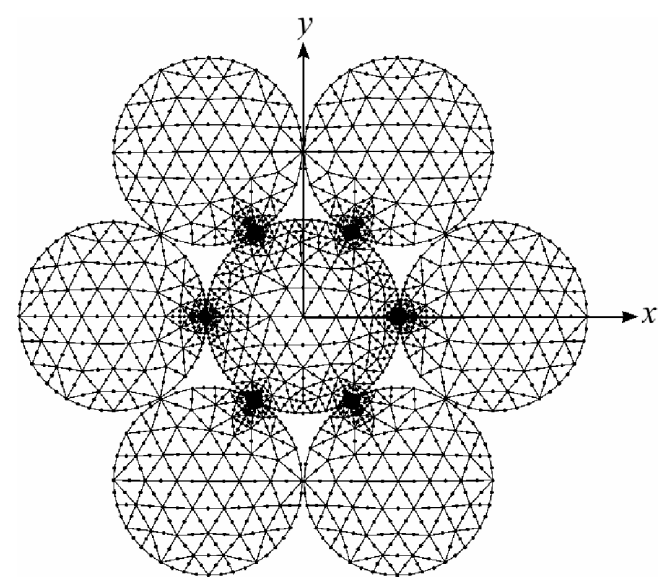

Figure 7: Cross-section FE mesh for the seven-wire strand $\phi=7.9^{\circ}$ (6975 dofs).

We first compute the dispersion curves for single constitutive wires. Figure 8 shows the frequency vs. wavenumber and energy velocity vs. frequency plots for a single core wire and for a single peripheral wire. As already shown in previous papers [21, 22], minor differences are found between cylindrical and helical wires for small lay angles ( $7.9^{\circ}$ being a small lay angle). A very small velocity decrease can be observed because waves travel at slower velocity in the curved wire. It should be noted that the shift observed for the $F(1,2)$ mode is indeed mainly due to the fact that the cross-section radius of a peripheral wire is slightly different from that of the central one. An interesting point to be outlined for the helical wire is that a strong decrease of energy velocity occurs for the $L(0,1)$ mode at lowest frequencies. This decrease is confirmed by experiments in the next subsection. Note that this phenomenon also occurs for the torsional $T(0,1)$ mode (see Fig. 8).

Figure 9 gives the frequency vs. wavenumber plot and the energy velocity plot for the seven-wire strand. Compared to Fig. 8, a far more complex 

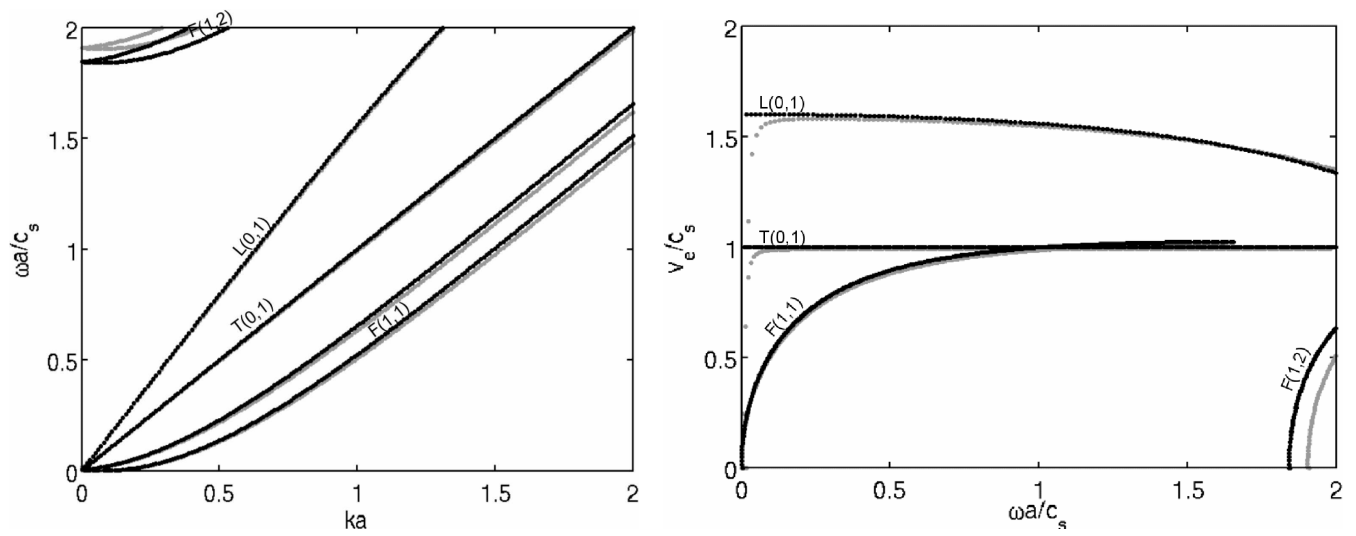

Figure 8: Dispersion curves for single wires. Black: central wire, gray: peripheral wire. Left: plot of normalized frequency vs. wavenumber, right: plot of normalized energy velocity vs. frequency.

behaviour is observed resulting from strong interactions between wires. It clearly shows an apparent cut-off of the fastest mode (compressional-like $L(0,1)$ mode) around $\omega a / c_{s}=0.35$, corresponding to $68 \mathrm{kHz}$. This phenomenon is also confirmed by experiments in the next subsection. In fact, this apparent cut-off is due to a rapid veering of branches occurring near 0.35 - this can be more clearly observed in the normalized frequency vs. wavenumber dispersion curves (see Fig. 9 - left). As a consequence, the upper curve of the energy velocity plot (Fig. 9 - right) is indeed composed of two distinct branches, describing the behaviour of two distinct wave modes.

Though somewhat subjective, a visual inspection of modeshapes has been performed in order to identify the evolution of both branches on a wider frequency range, as obtained in Fig. 10. The frequency vs. wavenumber plot exhibits a lower branch and an upper branch, denoted 1 and 2 respectively. As observed in the energy velocity plot (Fig. 10 - right), the fastest mode 

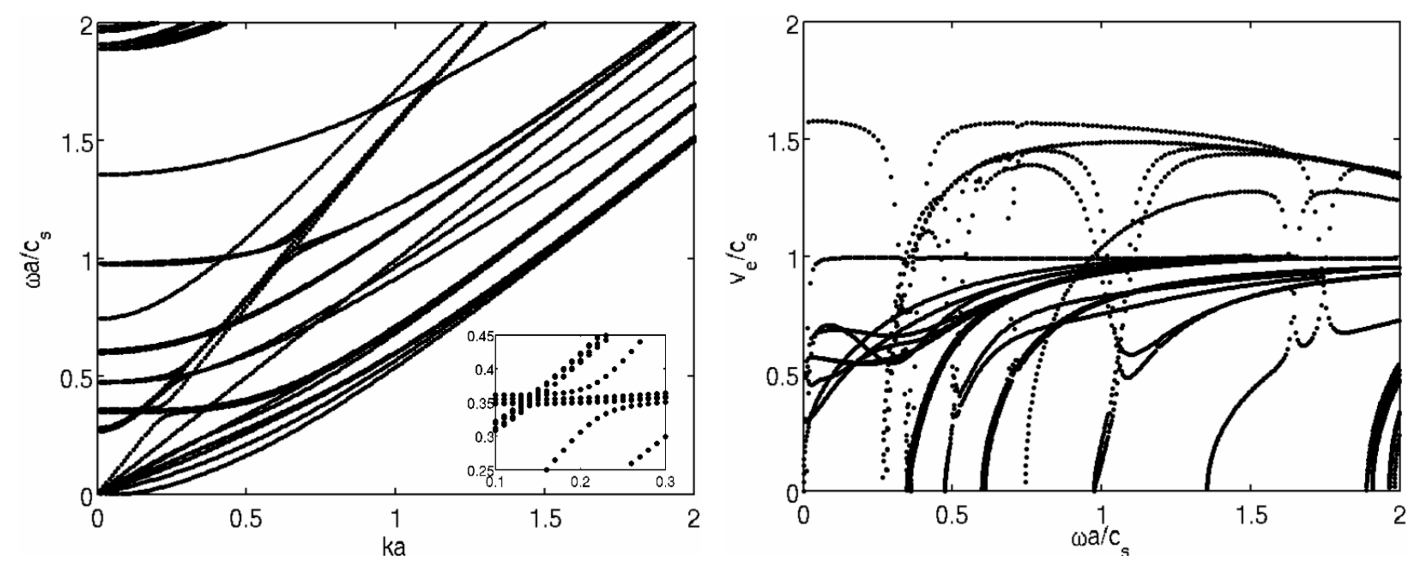

Figure 9: Dispersion curves for the seven-wire strand. Left: plot of normalized frequency vs. wavenumber (inset: zoom on the veering region of compressional-like $L(0,1)$ modes), right: plot of normalized energy velocity vs. frequency.

corresponds to branch 1 at low frequencies and branch 2 at higher frequencies. Comparing Figs. 8 and 10, it may be seen that the behaviour of branch 1 at low frequencies (1a portion) tends to the compressional $L(0,1)$ cylinder mode. Its group velocity then quickly drops around the cut-off frequency of branch 2 , near $\omega a / c_{s}=0.35$. Branch 1 then asymptotes to the flexural $F(1,1)$ cylinder mode (1c portion), while branch 2 becomes the $L(0,1)$ cylinder mode (2c portion). It must be emphasized that both branches asymptotes to the energy velocity of the $L(0,1)$ mode inside a single wire (of radius $a$ ) - see Figs. 8 and 10 - as opposed to a cylinder with an equivalent radius to the strand (of about $3 a$ ). Note that another veering happens to branch 2 around $\omega a / c_{s}=1.7$, where the energy velocity suddenly drops.

Surprisingly, a similar trend can be observed in pipes [30], where the $\mathrm{L}(0,1)$ and $\mathrm{L}(0,2)$ modes play the role of branches 1 and 2: in pipes, the 

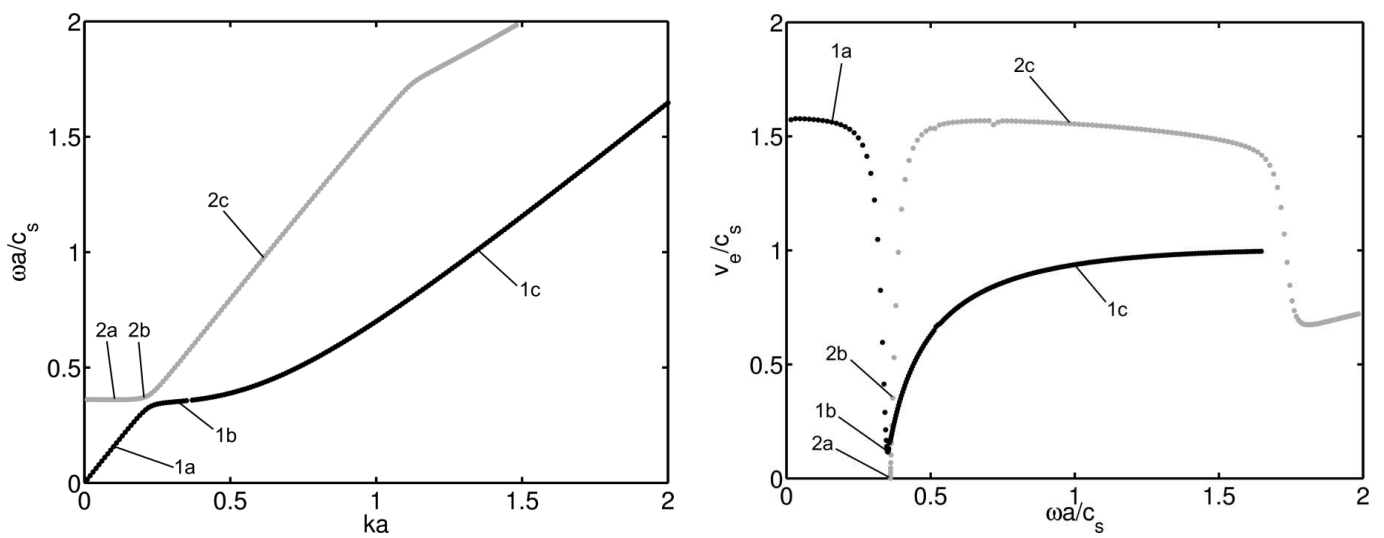

Figure 10: Branch identification of compressional-like $L(0,1)$ modes for the seven-wire strand (black: branch 1, gray: branch 2). Left: plot of normalized frequency vs. wavenumber. Right: plot of normalized energy velocity vs. frequency. The modeshapes corresponding to points $1 \mathrm{a}, 1 \mathrm{~b}$ and $1 \mathrm{c}$ are given by Fig. 11, those corresponding to $2 \mathrm{a}, 2 \mathrm{~b}$ and $2 \mathrm{c}$ are given by Fig. 12 .

$\mathrm{L}(0,1)$ mode at low frequencies is the extensional type mode (corresponding to S0 in a plate); its group velocity drops at around the cut-off frequency of the $\mathrm{L}(0,2)$ mode; the $\mathrm{L}(0,1)$ mode then asymptotes to the flexural type mode velocity (A0 in a plate), while the $\mathrm{L}(0,2)$ mode becomes the extensional-like mode.

The phenomenon related to branch 1 and branch 2 could also be interpreted as curve veering [31, 32] (repulsion of branches, veering away from each other instead of crossing), encountered in eigenvalue problems of weakly coupled systems. Both branches swap properties around $\omega a / c_{s}=0.35$ in a continuous but rapid way, and branch 2 seems to continue on the path previously followed by branch 1 , which is characteristic of curve veering phenomena.

Figure 11 shows the modeshape evolution of branch 1 computed at points 
1a, $1 \mathrm{~b}$ and 1c. These points are located in Fig. 10 and correspond to the solutions $\left(k a, \omega a / c_{s}, v_{e} / c_{s}\right)=(0.1,0.16,1.56),(0.31,0.35,0.12)$ and $(1.34,1.00,0.94)$ respectively. In this paper, modes are normalized with respect to the mass matrix M. Between points $1 \mathrm{a}$ and $1 \mathrm{~b}$ (in the veering zone), it can be seen that the modeshape undergoes significant transformations. At point 1c, it becomes more complex and exhibits a combined flexural-torsional motion. Figure 12 shows the modeshape evolution of branch 2, computed at points 2a, $2 \mathrm{~b}$ and 2c (located in Fig. 10). These points correspond to the solutions $\left(k a, \omega a / c_{s}, v_{e} / c_{s}\right)=(0.1,0.36,0.00),(0.2,0.37,0.35)$ and $(0.63,1.00,1.55)$ respectively. Comparing the modeshapes of points 1a and 2c, one can conclude that branch 1 and branch 2 have interchanged their shapes (this is another feature of veering phenomena). Their global axial motion confirms that they are compressional-like modes. However, these modes are not exactly similar because the real parts of their axial displacement have opposite signs.

Some computations have also been performed for a straight strand made of seven straight wires (results not shown for conciseness). Though dispersion curves are different from that of the helical strand, the veering phenomenon around 0.35 still exists in a straight strand. For a $7.9^{\circ}$ lay angle strand, we can conclude that the curvature of peripheral wires has a weak influence on this phenomenon compared to inter-wire contact effects.

\subsection{Experiments}

The final aim of this work is to perform a first validation of the seven-wire propagation model by comparing theoretical results to experiments in the low-frequency range for the compressional-like $L(0,1)$ modes. This was done on the basis of axisymmetric longitudinal guided waves measurements using 

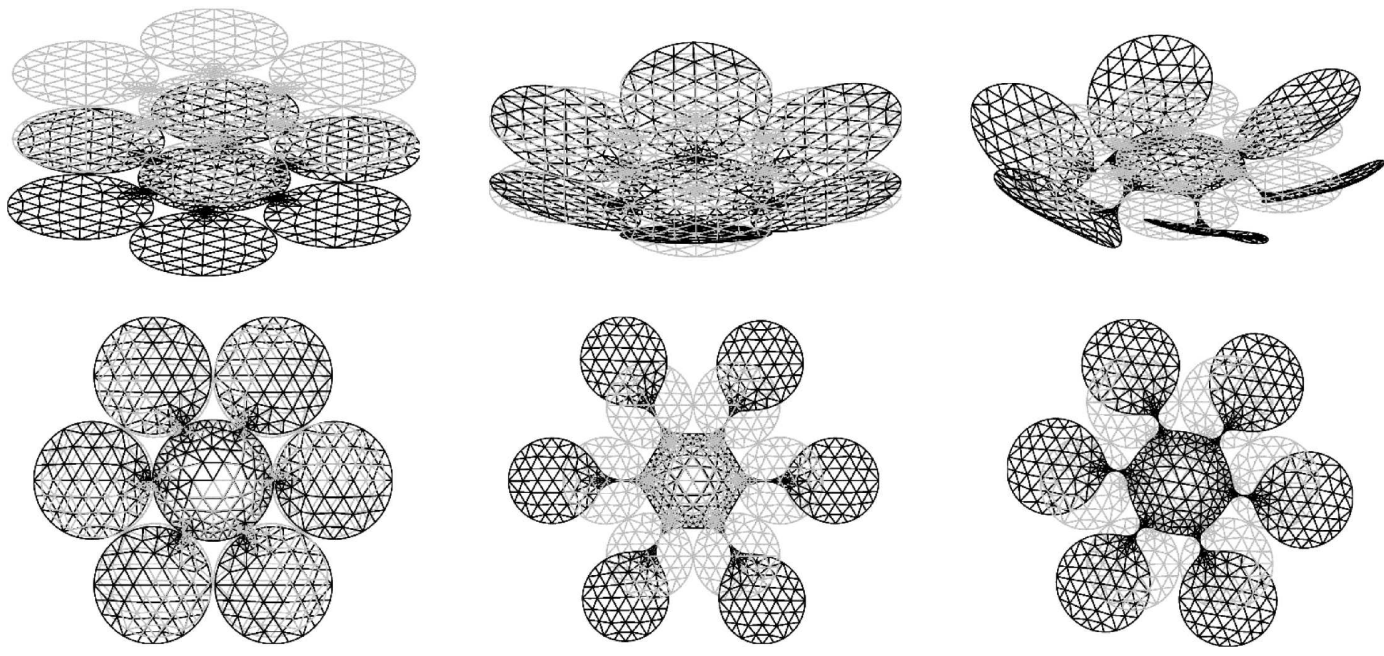

Figure 11: From left to right: real part (top) and imaginary part (bottom) of the displacement modeshape of branch 1 computed at points 1a, 1b and 1c (located in Fig. 10). Gray meshes correspond to the undeformed cross-section. The view of imaginary part is $2 \mathrm{D}$ for clarity. 

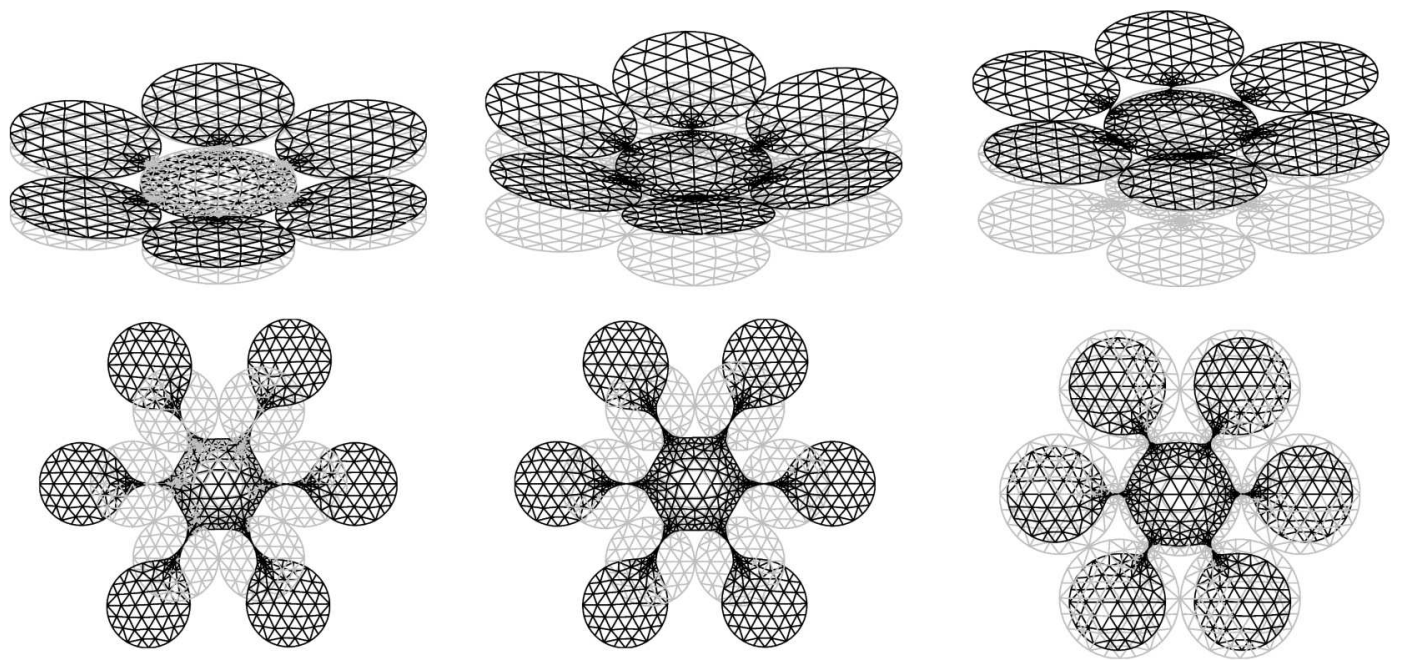

Figure 12: From left to right: real part (top) and imaginary part (bottom) of the displacement modeshape of branch 2 computed at points 2a, 2b and 2c (located in Fig. 10). Gray meshes correspond to the undeformed cross-section. The view of imaginary part is $2 \mathrm{D}$ for clarity. 
magnetostrictive transducers. Explanations on this experimental device can be found in Laguerre et al. [4, 5].

The device consists of two small encircling dynamic coil transducers, one transmitter and one receiver, each being mounted coaxially with a solenoid coil for the static longitudinal polarizing magnetic field. The time pulse excitation to the transmitting transducer is a low radio-frequency burst with a frequency bandwidth equal to its central frequency. The detected time signal is then processed using a short-time Fourier transform (spectrogram method) to recover the group velocity dispersion curves. The experimental group velocity dispersion curve is then deduced from the spectrogram by dividing the known travelling distance separating two arrivals with the associated group-time delay at each frequency.

A first step is devoted to the analysis of the individual constituting wires, that are the cylindrical core wire and the peripheral helical wire respectively. Figure 13 shows the comparison between measured and computed group velocities for the $L(0,1)$ mode of the single central cylindrical wire (radius of $2.7 \mathrm{~mm}$ ). For SAFE computations, note that group and energy velocities are equal because no damping was considered in this paper [28]. We observe a very good agreement between both curves, with the classical behaviour of the $L(0,1)$ mode group velocity, starting from the bar velocity estimated here at $5274 \mathrm{~m} . \mathrm{s}^{-1}$ and decreasing with frequency in this low-frequency range (less than $360 \mathrm{kHz}$ here).

The same procedure was applied to the measured time waveforms of the single peripheral helical wire (radius of $2.61 \mathrm{~mm}$, lay angle of $7.9^{\circ}$ ). In Fig. 14, the particular $L(0,1)$ low-frequency behaviour intrinsic to the helical geom- 


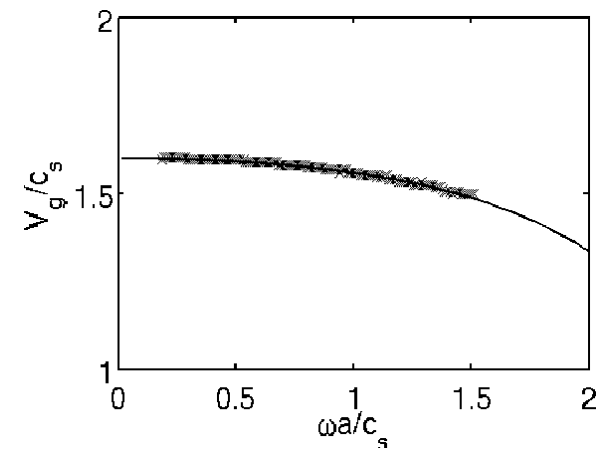

Figure 13: Central wire (cylindrical). Normalized group velocity vs. frequency measured with the magnetostrictive device (x-mark) and computed with the SAFE model (solid line) for the $L(0,1)$ mode.

etry described in the previous subsection is very well reproduced (low frequencies propagate much slower than higher frequencies). Note that such a velocity decrease is also in accordance with experimental observations of Kwun et al. [3].

Finally, the case of the seven-wire strand is considered. Numerical vs. experimental comparisons were performed by superimposing the SAFE computed group time-delays at each frequency upon the measured spectrograms. This only concerns the direct transmitter-to-receiver wave whose characteristics are not influenced by the strand-end reflections. A missing frequency band is observed in Fig. 15 in our experimental dataset. Such a missing band was initially experimentally found by Kwun et al. [3], and referred to as 'notch frequency'. This phenomenon is well reproduced by the sevenwire strand model, assuming stick contact conditions between constitutive wires. As explained in the previous subsection, this missing frequency band is related to curve veering between two distinct wave modes. The veering 


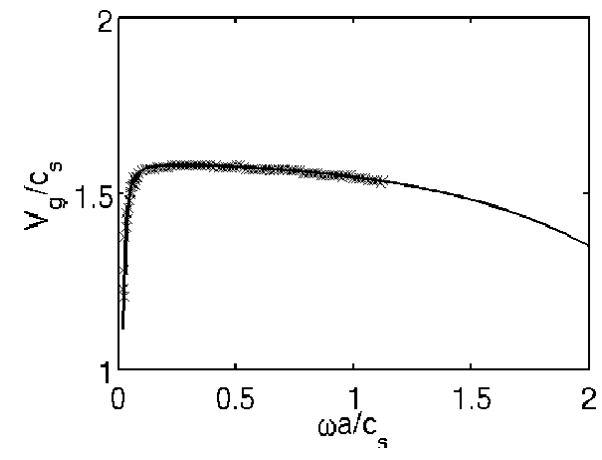

Figure 14: Peripheral wire (helical). Normalized group velocity vs. frequency measured with the magnetostrictive device (x-mark) and computed with the SAFE model (solid line) for the $L(0,1)$ mode.

central frequency of the seven-wire strand specimen used in this study is estimated at $67 \mathrm{kHz}$ from experimental data. This value was determined from the spectrum of the direct wave measured at different transmitter-to-receiver distances. The theoretical veering central frequency of $68 \mathrm{kHz}$ is hence in quite good agreement with experiments.

As a final remark, it should be noted that the 'notch frequency' found by Kwun et al. [3] in their experiments was around $80 \mathrm{kHz}$ at very low prestress, which is significantly greater than the one found in this paper. In fact, their strand had a nominal radius of $12.7 \mathrm{~mm}$ with a pitch of $22 \mathrm{~cm}$. This gives a lay angle of $6.9^{\circ}$ : as explained in the previous subsection, the difference with our lay angle of $7.9^{\circ}$ can be neglected. However, their core wire radius was $2.16 \mathrm{~mm}$ instead of $2.7 \mathrm{~mm}$ here, and their bar velocity was slightly lower. With $a=2.16 \mathrm{~mm}$ and $E=2.1 \mathrm{e}+11 \mathrm{~Pa}$, the normalized frequency of 0.35 found in this paper yields a satisfying result of $83 \mathrm{kHz}$. 


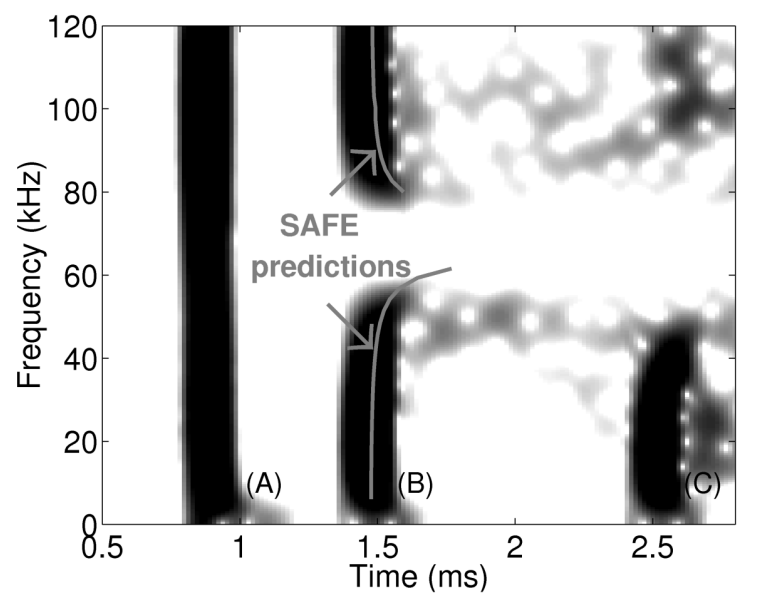

Figure 15: Seven-wire strand. SAFE predictions (gray solid lines) of group-time delays for the compressional-like $L(0,1)$ modes superimposed to the experimental spectrogram. (A), (B) and (C) respectively denote the arrivals related to the electro-magnetic coupling between coils, the direct mechanical wave and the first reflection of the direct wave.

\section{Conclusion}

In this paper, a SAFE method has been proposed to analyse elastic guided wave propagation along multi-wire helical strands. It is is based on a specific twisting coordinate system that can be considered as a special case of the helical system. This system allows to preserve translational invariance along the straight axis of multi-wire waveguides made of a central straight wire and several peripheral helical wires. This has been checked by comparing reference results for a single cylindrical waveguide and for a single helical waveguide with those computed for a strongly twisting coordinate system. A useful expression has also been proposed for computing the energy velocity in a straightforward way. Dispersion inside a typical seven-wire strand has then been investigated by assuming stick contact conditions and results have 
been compared to experimental tests.

From a physical point of view, a far more complex behaviour than for single wires has been observed. It has been found that the fastest compressionallike mode inside the seven-wire strand looks like the $L(0,1)$ one for a single wire, excepted in a frequency region typically around $68 \mathrm{kHz}$ where a quick drop of energy velocity occurs due to curve veering between two distinct modes. This value agrees well with experimental results obtained in this paper with encircling longitudinally-polarized magnetostrictive transducers, where curve veering manifests itself as a missing frequency band whose central frequency is around $67 \mathrm{kHz}$. Hence, the proposed seven-wire propagation model allows a relevant interpretation of this experimentally observed phenomenon, often referred to as 'notch frequency' in the literature. Further studies should deal with the effects of initial loading.

\section{Acknowledgements}

The authors wish to thank the anonymous reviewers for their helpul comments.

\section{References}

[1] T. R. Meeker, A. H. Meitzler, Guided wave propagation in elongated cylinders and plates, in: Physical Acoustics: Principles and Methods, mason Edition, Vol. 1, Academic, New York, 1964, pp. 111-167.

[2] J. Zemanek, An experimental and theoretical investigation of elastic wave propagation in a cylinder, Journal of the Acoustical Society of America 51 (1972) 265-283. 
[3] H. Kwun, K. A. Bartels, J. J. Hanley, Effects of tensile loading on the properties of elastic-wave propagation in a strand, Journal of the Acoustical Society of America 103 (1998) 3370-3375.

[4] L. Laguerre, M. Brissaud, J.-C. Aime, Low-frequency ultrasound reflectometry device based on magnetoelastic transducers for the non destructive evaluation of steel rods and cables, Bulletin des Laboratoires des Ponts et Chaussées 239 (2002) 7-27.

[5] L. Laguerre, M. Brissaud, J.-C. Aime, Magnetostrictive pulse-echo device for non-destructive evaluation of steel cylindrical materials using guide waves, Ultrasonics 39 (2002) 503-514.

[6] P. Rizzo, F. L. di Scalea, Load measurement and health monitoring in cable stays via guided wave magnetostrictive ultrasonics, Materials Evaluation 62 (2004) 1057-1065.

[7] P. Rizzo, F. L. di Scalea, Wave propagation in multi-wire strands by wavelet-based laser ultrasound, Experimental Mechanics 44 (2004) 407-415 .

[8] B. Pavlakovic, M. J. S. Lowe, P. Cawley, Inspection of tendons in post-tensioned concrete using guided ultrasonic waves, Insight: Nondetructive Testing and Condition Monitoring 41 (1999) 446-452.

[9] M. D. Beard, M. J. S. Lowe, P. Cawley, Ultrasonic guided waves for inspection of grouted tendons and bolts, Journal of Materials in Civil Engineering 212 (2003) 212-218. 
[10] L. Gavric, Computation of propagative waves in free rail using a finite element technique, Journal of Sound and Vibration 185 (1995) 531-543.

[11] T. Hayashi, W.-J. Song, J. L. Rose, Guided wave dispersion curves for a bar with an arbitrary cross-section, a rod and rail example, Ultrasonics 41 (2003) 175-183.

[12] V. Damljanovic, R. L. Weaver, Propagating and evanescent elastic waves in cylindrical waveguides of arbitrary cross-section, Journal of the Acoustical Society of America 115 (2004) 1572-1581.

[13] I. Bartoli, A. Marzani, F. L. di Scalea, E. Viola, Modeling wave propagation in damped waveguides of arbitrary cross-section, Journal of Sound and Vibration 295 (2006) 685-707.

[14] A. Demma, P. Cawley, M. Lowe, The effect of bends on the propagation of guided waves in pipes, Journal of Pressure Vessel Technology 127 (2005) 328-335.

[15] S. Finneveden, M. Fraggstedt, Waveguide finite element for curved structures, Journal of Sound and Vibration 312 (2008) 644-671.

[16] O. Onipede, S. B. Dong, Propagating waves and end modes in pretwisted beams, Journal of Sound and Vibration 195 (1996) 313-330.

[17] D. J. Mead, Wave propagation in continuous periodic structures: research contributions from southampton, 1964-1995, Journal of Sound and Vibration 190 (1996) 495-524. 
[18] D. J. Mead, A general theory of harmonic wave propagation in linear periodic systems with multiple coupling, Journal of Sound and Vibration 27 (1973) 235-260.

[19] L. Gry, C. Gontier, Dynamic modeling of railway track: a periodic model based on a generalized beam formulation, Journal of Sound and Vibration 199 (1997) 531-558.

[20] B. R. Mace, D. Duhamel, M. J. Brennan, Finite element prediction of wave motion in structural waveguides, Journal of the Acoustical Society of America 117 (2005) 2835-2843.

[21] F. Treyssède, Numerical investigation of elastic modes of propagation in helical waveguides, Journal of the Acoustical Society of America 121 (2007) 3398-3408.

[22] F. Treyssède, Elastic waves in helical waveguides, Wave Motion 121 (2008) 457-470.

[23] D. Chapelle, K. J. Bathe, The Finite Element Analysis of Shells - Fundamentals, Springer, 2003.

[24] A. Gray, E. Abbena, S. Salamon, Modern Differential Geometry of Curves and Surfaces with Mathematica, 3rd Edition, Chapman \& Hall, Boca Raton, 2006.

[25] A. Nicolet, F. Zola, S. Guenneau, Modeling of twisted optical waveguides with edge elements, The European Physical Journal Applied Physics 28 (2004) 153-157. 
[26] A. Nicolet, F. Zola, Finite element analysis of helicoidal waveguides, IET Science, Measurement and Technology 28 (2007) 67-70.

[27] F. Tisseur, K. Meerbergen, The quadratic eigenvalue problem, SIAM Review 43 (2001) 235-286.

[28] A. Bernard, M. J. S. Lowe, M. Deschamps, Guided waves energy velocity in absorbing and non-absorbing plates, Journal of the Acoustical Society of America 110 (2001) 186-196.

[29] J. D. Achenbach, Wave Propagation in Elastic Solids, North-Holland, Amsterdam, 1973.

[30] M. J. S. Lowe, D. N. Alleyne, P. Cawley, Defect detection in pipes using guided waves, Ultrasonics 36 (1998) 147-154.

[31] N. C. Perkins, C. D. Mote, Comments on curve veering in eigenvalue problems, Journal of Sound and Vibration 106 (1986) 451-463.

[32] C. Pierre, Mode localization and eigenvalue loci veering in disordered structures, Journal of Sound and Vibration 126 (1988) 485-502. 